\title{
O.S.P.
}

L'orientation scolaire et professionnelle

$30 / 3 \mid 2001$

Juger et être jugé : perspectives psychosociales

\section{A., Touraine, \& F., Khosrokhavar. La recherche de soi. Dialogue sur le sujet.}

Paris : Fayard.

Alain Baubion-Broye

(2) OpenEdition

Journals

Édition électronique

URL : http://journals.openedition.org/osp/5172

DOI : $10.4000 /$ osp. 5172

ISSN : 2104-3795

Éditeur

Institut national d'étude du travail et d'orientation professionnelle (INETOP)

Référence électronique

Alain Baubion-Broye, «A., Touraine, \& F., Khosrokhavar. La recherche de soi. Dialogue sur le sujet. », L'orientation scolaire et professionnelle [En ligne], 30/3 | 2001, mis en ligne le 20 juillet 2016, consulté le 16 décembre 2020. URL : http://journals.openedition.org/osp/5172 ; DOI : https://doi.org/10.4000/ osp.5172

Ce document a été généré automatiquement le 16 décembre 2020.

(c) Tous droits réservés 


\section{A., Touraine, \& F., Khosrokhavar. La recherche de soi. Dialogue sur le sujet.}

Paris : Fayard.

Alain Baubion-Broye

\section{RÉFÉRENCE}

Paris : Fayard.

1 Le dernier ouvrage de Touraine se présente comme un dialogue, retranscrit et retouché, avec son ancien élève, devenu l'un de ses collaborateurs, Farhad Khosrokhavar. Cet ouvrage, de plus de 300 pages, s'ouvre sur deux chapitres denses : « Invitation » (signé Touraine), « Introduction » (signé Farhad Khosrokhavar).

2 Au plus court, on pourrait observer que Touraine est, depuis quelque temps déjà, un sociologue de la subjectivité au moins parce qu'il veut résolument se désassujettir des sociologies du XIX' siècle, de leurs parentes et collatérales que le XX`a promues.

3 On retrouvera, dans ce dialogue, les grands thèmes qui mobilisent Touraine. Ils sont resitués dans sa propre aventure de chercheur et d'homme, à travers sa formation et dans l'expérience de rencontres décisives qui ont compté pour lui. Avec Labrousse et Le Goff (les historiens), avec Friedmann (son initiateur à la sociologie). Avec Aron (membre coupablement retors de son jury de thèse). Avec Chombart de Lauwe, Maucorps, Morin. Avec les ouvriers du Nord qu'il a cotoyés au quotidien de leur labeur, et dont la condition lui a, en large part, inspiré ses premières recherches sur « La conscience ouvrière ». Avec les intellectuels et les gens des peuples latino-américains. Avec le Chili, pays de son épouse, décédée il y a quelques années.

4 Le texte est souvent émouvant, lorsque Touraine évoque sans fard, ni blabla son intimité. L'homme est parfois un peu douloureux, parfois désenchanté ; il est peu complaisant avec lui-même : il se juge antipathique... Trop sérieux et trop raide pour 
partager le rire des autres, se croit imprécis, peu présent en amitié, sauf quand elle est professionnelle. Et puis, qu'en est-il aujourd'hui de sa croyance ancienne que le travail, la rédaction de livres, d'articles, devaient être mis au-dessus du reste de la vie ? Regrets voilés.

Nettes, gentiment fureteuses, rarement convenues, les questions de Farhad Khosrokhavar entraînent Touraine à livrer des réponses soucieuses de traduire les convictions et les hésitations qui continuent de marquer son parcours intellectuel, social, politique. Les questions de F. K., comme les réponses de T., ne sont pas exemptes de redondances ; elles donnent au dialogue son sens vécu et aux idées un caractère affectueusement partagé, une facture d'authenticité. Disons-le, sans finasser, les différents chapitres de l'ouvrage sont, à nos yeux, d'intérêt inégal. Peut-être parce que nous ne sommes pas suffisamment proches de ses affinités politiques ni preneurs de ses analyses prospectives. Le risque, au demeurant mineur, que doit assumer tout auteur qui se dévoile ainsi, est de ne pas être suivi par ses lecteurs. Peut-être aussi avons-nous eu tendance à rechercher et donc à retenir dans son propos ce que nous savions déjà par ses travaux antérieurs : sur le mouvement social, sur l'acteur..., et avons-nous, à l'inverse, été trop attentifs à ses « oublis ». Peut-être, surtout, en mauvaise obstination, avons-nous tenté de scruter, dans ses orientations actuelles, des réponses aux questions critiques et respectueuses que nous lui posions, jadis, en 1989 (cf. "Dynamiques sociales et changements personnels ", Édit. du C.N.R.S.)... Des questions qu'il n'a probablement jamais lues mais auxquelles, en certains passages de l'ouvrage, on s'est plu à chercher des réponses partielles.

6 On pourra regretter que Touraine ne se confronte pas à d'autres : ses homologues, les sociologues, dont les positions révèlent des liens ténus, des affinités plus ou moins militantes avec "les mouvements historiques " (notamment, dans le procès de globalisation) avec « la colère " (qui prend pour Touraine une sorte de statut sociopsychologique, cf. p. 172) ou avec la « misère du monde »), bref avec tout ce qui suscite les exigences de justice, de droit, de dignité et la conscience résistante et réflexive, caractéristiques du sujet tourainien. On pourrait aussi s'étonner que pas plus la psychologie que la psychanalyse ne paraissent beaucoup préoccuper la recherche de Touraine (cf. les chapitres " sur la science sociale ", « sur le sujet comme rapport à soi »). Et pourtant... selon lui, pour que le sujet se construise et existe il faut qu'il y ait conflictualité et individuation, qu'il y ait la reconnaissance d'autrui comme sujet, il faut « la création d'un espace institutionnel démocratique, un espace de droit et de garanties " (p. 149 et p. 173). C'est sur les vestiges ou les limites du mouvement social et dans la mutation d'un acteur de "moins en moins social » (p. 9) que le sujet se promeut en rupture ou opposition, qu'il produit du sens, qu'il s'affirme par sa singularité et sa liberté. L'insistance de Farhad Khosrokhavar pour évoquer des contextes sociaux où l'individu est englué dans des contraintes qui le tirent vers la "désubjectivation " n'arrive pas à ébranler l'auteur (et le citoyen) Touraine dans la certitude qu'aucune situation, fût-elle aversive, privative, aliénante, ne peut interdire à l'individu de devenir sujet, de se faire reconnaître comme tel, parmi et contre d'autres, de désirer avoir prise sur ses milieux et sa vie. La visée d'unité est, en quelque sorte, au coeur du sujet. " Être sujet c'est sentir l'unité du Je au-delà de la diversité des expériences » (p. 230). Et c'est autant la capacité qu'il détient de rassembler des ressources internes contre la raison instrumentale, "contre les risques que lui font encourir la loi du marché » (p. 144) et les dogmes des communautarismes reclus. 
7 Cette visée et cette capacité du sujet se soutiennent de sentiments et d'affects. Dans ce sujet, il y a de la joie ! « Il y a la joie de la rencontre avec l'autre sujet... La joie que procure la reconnaissance en droit de tous et toutes à être sujets » (p. 248).

En réalité, même s'il est souvent menacé d'être défait ou détruit, le sujet demeure force de dépassement et prise de distance à l'égard de soi. Son autonomie créatrice (instituante) face aux institués est construite et se déploie au travers des écarts qui caractérisent les rapports de soi à soi et la relation interpersonnelle, qu'aiguillonnent l'inquiétude et l'incertitude qu'aucun " garant méta-social » ne régule et ne dompte de l'extérieur (cf. par exemple les chapitres 5 et 6 de l'ouvrage). Quelques ambiguïtés sinon des paradoxes - dont, selon nous, la pensée de Touraine s'accommode, existent dans son propos, quant à la genèse et à la structure de ce sujet, un peu transcendant... D'où vient que le sujet de Touraine puisse dire "Je ", qu'il puisse dire " non ", qu'il puisse communiquer, souffrir, être gai, amoureux, solidaire, produit et producteur de conflits ? D'où vient qu'il résiste, qu'il s'éprouve en principe d'unité ? Comment expliquer et justifier qu'il soit déclaré «non social, non psychologique » (p. 285) ? De la conflictualité qu'affronte son sujet, Touraine n'explore, ni sous forme hypothétique ni empiriquement, les origines, les conditions, le rôle dans ses développements personnels et ses relations intersubjectives. Le retour de ce sujet sur son " intérieur » n'est-il pas l'une des figures post-modernes de la solitude, mais également une aliénation ? Le " versant optimiste " de la pensée de Touraine regimbe devant ce type de question, puisque, pour Touraine, ce retour semble être un détour au cours duquel le sujet découvre de nouvelles formes d'action et de mobilisation (en particulier culturelles), modifie les rapports entre vie privée et vie publique, entre l'individuel et le collectif.

Qu'en est-il des processus de subjectivation ? Pour Touraine, la subjectivation passe par la perte de soi. Ce qui confine au danger de " jeter le je avec le moi ». "Elle consiste, écrit-il, à transformer en sujet des expériences de routine, de passivité ou, au contraire, d'exaltation... [la séparation de soi que le sujet vit] doit conduire à revenir à soi, à ne pas perdre conscience de soi » (p. 125). À l'évidence, le sujet de Touraine n'est pas tout à fait de la même étoffe que celui d'Ehrenberg... Bien que Touraine compatisse avec les difficultés d'être soi du sujet dépressif, pour lui, l'issue de ces difficultés n'est pas seulement la dépression qui serait plutôt l'apanage des anxieux narcissiques, socialement intégrés... Et, pourrait-on dire, de ceux qui voudraient bravement fonctionner à " l'intemalité » mais qui en seraient, hélas, empêchés.

On l'aura, bien sûr, compris, dans cette dernière publication de Touraine - qui doit beaucoup à Farhad Khosrokhavar - on trouve matière à discussions, à interrogations et à étonnements, cela même à quoi, sans cesser de séduire, son oeuvre importante nous a accoutumés. 